\title{
Pós-alta em hanseníase: uma revisão sobre qualidade de vida e conceito de cura
}

\author{
Mara Dayane Alves Ribeiro¹, Sabrynna Brito Oliveira², Marcelo Carvalho Filgueiras ${ }^{3}$
}

\begin{abstract}
RESUMO
A fragilidade do acesso à atenção, a estigmatização e a desinformação tornam o período pós-alta em hanseníase dificultoso para os indivíduos afetados. OBJETIVO: Explorar o momento pós-alta de hanseníase, na busca de ações mais integrais aos portadores, e ampliação do conceito de cura da doença. METODOLOGIA: Busca nos bancos de dados: BVS, SCIELO, PUBMED, LILACS, BIREME e Google Acadêmico. Os termos utilizados foram: hanseníase, pós-alta, sequelas, estigma e qualidade de vida. RESULTADOS: Selecionou-se 44 artigos, datados de 1995 a 2012. Foi observada a necessidade de políticas específicas para a abordagem em pacientes que receberam alta por cura. É demonstrada influência negativa na Qualidade de Vida (QV) pelos aspectos socioculturais que envolvem a doença, assim como, pelas deformidades físicas. CONCLUSÕES: A adoção de intervenções específicas à população em pós-alta de hanseníase é indispensável para o cumprimento de um dos princípios norteadores do Sistema Único de Saúde, o de integralidade.
\end{abstract}

Descritores: Hanseníase; Pós-alta; Qualidade de Vida; Estigma.

\section{Post treatment in Leprosy: a review about quality of life and cure concept}

\begin{abstract}
The fragility of access to care, stigma and misinformation makes the post treatment in Leprosy cornered for affected. OBJECT: To explore the post treatment in Leprosy seeking more whole shares to holders and expansion of the concept of cure. METHODS: Search in databases: BVS, SCIELO, PUBMED, LILACS, BIREME and google scholar. The used terms were: leprosy, post-high, sequela, stigma, quality of life. RESULTS: Were selected 44 articles, dating from 1995 to 2012. The need for specific policies to address in patients who were discharged after cure was observed. It is demonstrated negative influence on quality of life (QOL) by socio-cultural aspects surrounding the disease, as well as by physical deformities. CONCLUSIONS: The adoption of specific interventions to the population post treatment in Leprosy is essential to the accomplishment of one of the guiding principles of the Health System, the integrality.
\end{abstract}

Descriptors: Leprosy; Post-high; Quality of Life; Stigma.

${ }^{1}$ Fisioterapeuta graduada pela Universidade Federal do Piauí (UFPI), Parnaíba, PI, Brasil.

${ }^{2}$ Biomédica graduada pela Universidade Federal do Piauí (UFPI), Parnaíba, PI, Brasil.

${ }^{3}$ Mestre em Educação em Saúde pela Universidade de Fortaleza (UNIFOR), Fortaleza, CE, Brasil. 


\section{Introdução}

A hanseníase é uma doença crônica, infectocontagiosa, de evolução lenta, que possui como sinais e sintomas dermatoneurológicos lesões na pele e nos nervos periféricos, principalmente nos olhos, mãos e pés. 0 agente etiológico é o Mycobacterium leprae ou bacilo de Hansen, um parasita intracelular, que apresenta incubação nos macrófagos e tropismo por células cutâneas e células de nervos periféricos podendo se multiplicar. A doença é associada a danos de ordem psicológica e social, representando um grave problema de saúde pública mundial. ${ }^{1,2}$

A transmissão da doença se faz de forma direta, por via respiratória, através do contato com uma pessoa doente, sem tratamento, que elimina o bacilo para o meio exterior infectando outras pessoas suscetíveis. ${ }^{1} \mathrm{O}$ diagnóstico e 0 tratamento precoce dos casos são as medidas mais eficazes para se prevenir as incapacidades decorrentes da doença. A patologia e as deformidades a ela associadas são responsáveis pelo estigma social e pela discriminação aos pacientes e suas famílias. ${ }^{3,4}$

Devido ao poder incapacitante da doença associado ao estigma social, a hanseníase foi considerada problema de saúde pública pela Organização Mundial de Saúde (OMS), que passou a definir, a partir do ano 2000, metas para que os países chegassem a taxa estabelecida de menos de 1 caso por 10.000 habitantes. ${ }^{5,6}$ Martelli ${ }^{7}$, ressalta a necessidade de investir na produção de conhecimentos de áreas básica e aplicada que viabilizem uma maior compreensão dos mecanismos de transmissão da infecção, da efetividade dos métodos de prevenção e controle, como estratégias essenciais na "erradicação" da infecção pelo M. leprae.

Embora, o Brasil registre decréscimos contínuos nos coeficientes de prevalência e de detecção de casos novos de hanseníase, ainda é o segundo país com maior número de casos a nível mundial. As regiões Norte, Centro-Oeste e Nordeste são consideradas as mais endêmicas, com áreas de importante manutenção da transmissão. ${ }^{8,3}$ Mesmo com a decaída dos coeficientes, em 2010, o Brasil ainda apresentou prevalência média de 1,56 casos para cada 10.000 habitantes, correspondendo a 29.761 casos em tratamento. Neste mesmo ano, o país detectou 34.894 casos novos de hanseníase, correspondendo a um coeficiente de detecção geral de 18,2/100.000 habitantes.

No Ceará, Nordeste do Brasil, 7 municípios são considerados prioritários na eliminação da hanseníase: Caucaia, Crato, Fortaleza, Iguatu, Juazeiro do Norte, Maracanaú e Sobral. Considera-se que mais de 50\% da população do estado vive em municípios com prevalência superior a 5 casos/10 mil hab., enquanto a taxa ideal é menos de 1 caso/10 mil hab.6

As incapacidades e deformidades ocasionadas pela hanseníase podem acarretar problemas de ordem psicossocial, tais como, diminuição da capacidade de trabalho, limitação da vida social e depressão. Sendo responsáveis, também, pelo estigma e preconceito contra a doença, por ser uma enfermidade que se expressa nas partes externas do corpo.9,4,10

Um conjunto de características como crenças, medos, preconceitos e sentimento de exclusão atinge os portadores dessa doença e se efetiva a partir do isolamento social que os envolve. Estas pessoas preferem manterem-se calados a respeito do diagnóstico e ocultar seu corpo, na tentativa de esconder a doença, para evitar a rejeição e o abandono da sociedade. .11,12 $^{2}$

O momento pós-alta ocorre quando o indivíduo recebe alta por cura após ter concluído o tratamento poliquimioterápico (PQT) com êxito. Mesmo depois de curados, os indivíduos podem apresentar reações hansênicas (RH), (reações do sistema imunológico do doente ao Mycobacterium leprae) e recidivas (após completo a PQT, o indivíduo curado desenvolve novos sinais e sintomas da doença) que se apresentam através de episódios inflamatórios agudos e subagudos, acometendo tanto os casos Paucibacilares como os Multibacilares. As RH se apresentam como a principal causa de lesões dos nervos e de incapacidades provocadas pela hanseníase representando um grande problema no tratamento dos portadores. ${ }^{1,3}$

A fragilidade do acesso à atenção, os preconceitos, a auto-segregação e a desinformação tornam o período de pósalta dificultoso para os indivíduos afetados pela hanseníase, exigindo uma atenção de saúde longitudinal ao portador. 13,14

Este artigo tem por objetivo discutir o momento pós-alta de hanseníase, na busca de ações mais integrais aos portadores e ampliação do conceito de cura da doença. Pretendendo-se destacar o período depois da alta por cura em hanseníase como importante na continuidade da atenção à saúde ao portador desta patologia.

\section{Materiais e métodos}

Este estudo trata-se de uma revisão bibliográfica cuja busca foi realizada nos bancos de dados online BVS (Biblioteca Virtual de Saúde), SCIELO (Scientific Electronic Library Online), PUBMED, LILACS, BIREME e Google Acadêmico. Os 
termos utilizados foram: hanseníase, pós-alta, sequelas, estigma, qualidade de vida e seus correspondentes na língua inglesa.

Foram encontrados variados trabalhos que abordavam itens da temática desta revisão. Como critério de inclusão cada qual deveria tratar do período de pós-alta em hanseníase, alta medicamentosa, qualidade de vida e cuidado longitudinal de saúde para estas pessoas, sendo selecionados 44 artigos para integrar a presente revisão, datados de 1995 a 2012. Os artigos foram analisados individualmente tanto no seu conteúdo quanto no seu referencial teórico com a finalidade de abranger o universo da temática abordada e facilitar o acesso a artigos utilizáveis. Nesta pesquisa não houve restrição quanto à língua, sendo incluídos artigos na língua portuguesa, inglesa.

\section{Resultados e discussão}

Observou-se na literatura (tabela 1), a importância e a necessidade de políticas específicas para a abordagem em pacientes que receberam alta por cura de hanseníase. É necessária uma assistência integral da saúde abrangendo as sequelas que permanecem nestas pessoas mesmo após a alta, adotando-se um novo conceito de cura que vai além da eliminação do bacilo, um atendimento permanente visando à eliminação do estigma pelo qual passam estes indivíduos. ${ }^{4,16,17}$

Quando analisada a Qualidade de vida (QV) em portadores de hanseníase, estudos demonstram a influência negativa dos aspectos socioculturais que envolvem a doença, assim como, das deformidades físicas que se desenvolvem gerando desordens biopsicossociais nestes indivíduos (tabela 2).

Não foram encontradas propostas concretas para a continuação do cuidado em pós-alta nesta população, as ações contemplam o acompanhamento para as possíveis intercorrências: $\mathrm{RH}$ e recidivas. Destaca-se na literatura, a necessidade de protocolos que configurem ações mais globais que garantam também a cura das questões e danos subjetivos causados pela Hanseníase, exclusão social, estigma, auto-segregação.

Tabela 1 - Estudos ressaltando a necessidade de uma abordagem longitudinal de saúde para indivíduos em pós-alta de Hanseníase

\begin{tabular}{|c|c|c|c|}
\hline Autor & Amostra & Resultados encontrados & Conclusões \\
\hline Barbosa $^{14}$ & $\begin{array}{l}304 \text { afetados residentes em dois } \\
\text { municípios cearenses. }\end{array}$ & $\begin{array}{c}\text { Fragilidade de ações do programa de } \\
\text { controle da Hanseníase no momento } \\
\text { pós-alta em Sobral e Fortaleza. }\end{array}$ & $\begin{array}{c}\text { Necessidade de protocolos para } \\
\text { pós-alta em todos os níveis de } \\
\text { gestão. }\end{array}$ \\
\hline Ikehara $^{17}$ & $\begin{array}{l}\text { Pacientes tratados entre } 2007 \text { e } 2009 \text {, } \\
\text { em São José do Rio Preto-SP. Uso da } \\
\text { SALSA* e GI-OMS** para correlação }^{*} \text { de limitação e incapacidade. }\end{array}$ & $\begin{array}{c}\text { Da amostra ( } \mathrm{n}=54), 57,4 \% \text { refere } \\
\text { alguma limitação às atividades } \\
\text { (SALSA), 68,5\% possuíam algum grau } \\
\text { GI-OMS. }\end{array}$ & $\begin{array}{l}\text { Defendem que a atenção e o } \\
\text { cuidado ao paciente para evitar } \\
\text { sequelas deve ser contínuo, e não } \\
\text { necessariamente interrompido no } \\
\text { ato da alta medicamentosa. }\end{array}$ \\
\hline Martins $^{4}$ & $\begin{array}{c}\text { Utilizou a história oral de } 3 \text { mulheres } \\
\text { moradoras do Hospital Santa } \\
\text { Teresa, localizado em São Pedro } \\
\text { de Alcântara-SC, sobre o estigma, } \\
\text { preconceito e exclusão. }\end{array}$ & \begin{tabular}{|c|} 
Observa-se o afastamento das \\
pessoas menos esclarecidas e a \\
busca pelo modelo de aceitação \\
social. Além, de ser reconhecido como \\
diferente e não como discriminado.
\end{tabular} & $\begin{array}{c}\text { Presença de sequelas } \\
\text { permanentes que seguem mesmo } \\
\text { com a alta. }\end{array}$ \\
\hline Videres $^{15}$ & $\begin{array}{l}12 \text { ex-portadores de hanseníase } \\
\text { que foram asilados em Natal-RN. A } \\
\text { coleta de dados foi realizada com } \\
\text { entrevista semiestruturada, gravada } \\
\text { sobre exclusão social e, estigma e } \\
\text { preconceito. }\end{array}$ & \begin{tabular}{|} 
Relatos marcantes de sofrimento, \\
negação, rejeição, revolta que \\
repercute até após o término do \\
tratamento, interferindo negativamente \\
na reintegração social e familiar \\
desses indivíduos.
\end{tabular} & $\begin{array}{c}\text { Necessidade de os gestores e } \\
\text { profissionais locais da saúde } \\
\text { repensarem as estratégias } \\
\text { vigentes de reabilitação social } \\
\text { do doente e ex-doente de } \\
\text { hanseníase, visando à supressão } \\
\text { de uma estigmatização injusta e } \\
\text { nociva. }\end{array}$ \\
\hline
\end{tabular}




\begin{tabular}{|c|c|c|c|}
\hline Autor & Amostra & Resultados encontrados & Conclusões \\
\hline Lira $^{10}$ & $\begin{array}{l}\text { Descrição das atuais estratégias } \\
\text { de controle da hanseníase como } \\
\text { problema de saúde pública e } \\
\text { construção sociocultural da doença } \\
\text { pela humanidade }\end{array}$ & $\begin{array}{l}\text { Representação da doença como } \\
\text { um problema que gera sofrimento } \\
\text { social, centrado na cronicidade, } \\
\text { desmoralização, estigma e vergonha. }\end{array}$ & $\begin{array}{l}\text { Apontam a atenção ao enfermo } \\
\text { e o empowerment, inclusive no } \\
\text { pós-eliminação da hanseníase, } \\
\text { como tarefa essencial para a } \\
\text { naturalização da doença. }\end{array}$ \\
\hline Aquino $^{18}$ & $\begin{array}{l}\text { Análise de } 214 \text { prontuários de } \\
\text { pacientes portadores das diversas } \\
\text { formas clínicas de hanseníase, } \\
\text { atendidos na unidade de saúde da } \\
\text { Universidade Federal do Maranhão. }\end{array}$ & $\begin{array}{l}\text { Programa de controle da Hanseníase } \\
\text { no município de Buriticupu-MA } \\
\text { considerado precário. }\end{array}$ & $\begin{array}{c}\text { • Avaliação do programa } \\
\text { fundamental para o sucesso do } \\
\text { mesmo. } \\
\text { - Importância de examinar todos } \\
\text { os pacientes na alta. }\end{array}$ \\
\hline Ramos $^{19}$ & $\begin{array}{l}\text { Estimou-se a prevalência das } \\
\text { incapacidades em } 169 \text { pacientes } \\
\text { com qualquer das formas clínicas de } \\
\text { hanseníase no município de Várzea } \\
\text { Grande, MT, que já haviam concluído } \\
\text { tratamento nos anos anteriores. }\end{array}$ & $\begin{array}{c}50,2 \% \text { da amostra no estudo } \\
\text { apresentaram piora evolutiva da } \\
\text { sensibilidade, sendo esta piora } \\
\text { associada à presença de lesão neural } \\
\text { no momento do diagnóstico. }\end{array}$ & $\begin{array}{l}\text { Importância de monitorar a função } \\
\text { neural dos pacientes, muito } \\
\text { além do momento de finalizar a } \\
\text { PQT com o intuito de prevenir a } \\
\text { aparição de incapacidades em } \\
\text { pacientes que já receberam alta. }\end{array}$ \\
\hline Lustosa $^{16}$ & $\begin{array}{c}\text { Estudo observacional com } 107 \\
\text { pacientes } \\
\text { com a aplicação do SF-36 } \\
\text { delineando o perfil sociodemográfico, } \\
\text { clínico e epidemiológico dos } \\
\text { entrevistados. }\end{array}$ & $\begin{array}{l}\text { A hanseníase continua provocando } \\
\text { elevados níveis de lesões secundárias } \\
\text { que comprometem a capacidade de } \\
\text { trabalho e a qualidade de vida dos } \\
\text { acometidos, perpetuando o estigma } \\
\text { associado à doença. }\end{array}$ & $\begin{array}{l}\text { Reforça a necessidade de } \\
\text { estratégias mais efetivas de } \\
\text { controle da doença. Formas } \\
\text { graves e incapacitantes de } \\
\text { hanseníase está diretamente } \\
\text { relacionada à baixa QVRS }{ }^{* *} \text {, } \\
\text { mesmo no paciente curado. }\end{array}$ \\
\hline Souza ${ }^{20}$ & $\begin{array}{l}\text { Estudo retrospectivo com objetivo } \\
\text { de verificar a frequência de reações } \\
\text { hansênicas em pacientes com alta por } \\
\text { cura pela PQT. }\end{array}$ & $\begin{array}{l}\text { Pacientes que apresentaram } \\
\text { reações durante o tratamento, 35,5\% } \\
\text { continuaram pós-PQT. }\end{array}$ & $\begin{array}{l}\text { Existe relação diretamente } \\
\text { proporcional entre presença de } \\
\text { reações durante e após } \\
\text { o tratamento. }\end{array}$ \\
\hline Rodrigues $^{21}$ & $\begin{array}{l}\text { Revisados prontuários de } 149 \\
\text { pacientes que receberam alta } \\
\text { de hanseníase de } 1994 \text { a 1999, } \\
\text { utilizando a Ficha de Investigação de } \\
\text { Intercorrências Pós-Alta por Cura do } \\
\text { Ministério da Saúde. }\end{array}$ & $\begin{array}{l}23 \% \text { da amostra }(n=34) \text { apresentaram } \\
\text { reações hansênicas pós-alta. }\end{array}$ & $\begin{array}{c}\text { Necessidade de programas de } \\
\text { controle específicos para esse } \\
\text { novo grupo de pacientes em alta } \\
\text { por cura. }\end{array}$ \\
\hline
\end{tabular}

* Screening of Activity Limitation and Safety Awareness

** Grau de Incapacidade segundo a Organização Mundial de Saúde

*** Qualidade de Vida Relacionada à Saúde. 
Tabela 2 - Pesquisas observando Qualidade de Vida (QV) em portadores de hanseníase.

\begin{tabular}{|c|c|c|c|}
\hline Autor & Amostra & Resultados encontrados & Conclusões \\
\hline Savassi $i^{22}$ & $\begin{array}{l}32 \text { pacientes e seus respectivos } \\
\text { cuidadores domiciliares Aplicou-se } \\
\text { o Minimental, e os questionários } \\
\text { de avaliação de atividades de vida } \\
\text { a pacientes, e o WHOQoL-breve a } \\
\text { pacientes e cuidadores. }\end{array}$ & $\begin{array}{c}\text { A pesquisa demonstrou piores } \\
\text { escores de QV nos domínios físico } \\
\text { e mental, com melhores resultados } \\
\text { para o domínio social do WHOQOL*. } \\
\text { As peculiaridades do isolamento e do } \\
\text { estigma foram determinantes na QV. }\end{array}$ & $\begin{array}{l}\text { Escores de QV são influenciados } \\
\text { diretamente pelas sequelas e } \\
\text { evolução natural da hanseníase, } \\
\text { tendo relevância as reações } \\
\text { sociais à doença. }\end{array}$ \\
\hline Martins $^{23}$ & $\begin{array}{c}30 \text { portadores de hanseníase de } \\
\text { ambos os sexos, acima de } 18 \text { anos, } \\
\text { utilizando o SF-36 e um Questionário } \\
\text { Sócio-Demográfico-Ocupacional/ } \\
\text { QSDO }\end{array}$ & $\begin{array}{c}\text { Ressalta domínios na QV de } \\
\text { hansenianos: preconceito e estigma, } \\
\text { problemas culturais e sociais, apesar } \\
\text { da melhora dos aspectos médicos da } \\
\text { doença. }\end{array}$ & $\begin{array}{l}\text { Avaliar a QV de hansenianos } \\
\text { mostra-se de fundamental } \\
\text { importância para o } \\
\text { desenvolvimento de políticas } \\
\text { públicas para a promoção de } \\
\text { saúde e prevenção da doença } \\
\text { que repercutam positivamente na } \\
\text { esfera psico-sócio-cultural. }\end{array}$ \\
\hline Paschoal ${ }^{24}$ & $\begin{array}{c}28 \text { pacientes adultos de } \\
\text { ambos os sexos, em estado reacional. } \\
\text { Foram utilizados uma entrevista } \\
\text { e um roteiro sobre as } \\
\text { mudanças experimentadas pelo } \\
\text { cliente em } \\
\text { decorrência à crise reacional. }\end{array}$ & $\begin{array}{l}\text { Verificou-se que as crises } \\
\text { reacionais alteram consideravelmente } \\
\text { a QV do portador de hanseníase. } \\
\text { Encontraram-se dificuldades para lidar } \\
\text { com a dor, com as expectativas de } \\
\text { sonho e de futuro, com a alimentação } \\
\text { e o sono, entre outras, do cotidiano. }\end{array}$ & $\begin{array}{c}\text { Ressalta a análise dos } \\
\text { componentes comprometidos na } \\
\text { QV de hansenianos, como forma } \\
\text { de oferecer subsídios para uma } \\
\text { assistência mais eficaz, voltada } \\
\text { para as necessidades individuais } \\
\text { deste paciente. }\end{array}$ \\
\hline Joseph ${ }^{25}$ & $\begin{array}{c}30 \text { homens e } \\
20 \text { mulheres com idades entre } 15 \pm \\
70 \text { anos, em pós-alta por Hanseníase } \\
\text { analisados por meio do WHOQoL- } \\
\text { breve. }\end{array}$ & $\begin{array}{l}\text { Aponta as deformidades, assim como } \\
\text { a visibilidade destas, com relação } \\
\text { inversamente proporcional a QV, } \\
\text { enquanto a situação econômica } \\
\text { apresentou-se diretamente } \\
\text { relacionada a esta. }\end{array}$ & $\begin{array}{l}\text { QV decresce progressivamente } \\
\text { com a doença. }\end{array}$ \\
\hline Browers ${ }^{26}$ & $\begin{array}{l}100 \text { pessoas com incapacidades } \\
\text { relacionadas à hanseníase foram } \\
\text { avaliadas através do WHOQoL-breve. }\end{array}$ & $\begin{array}{l}\text { Sustento da família, satisfação com a } \\
\text { saúde, sexo, e limitações funcionais } \\
\text { mostraram associação significante } \\
\text { com baixos escores de QV. }\end{array}$ & $\begin{array}{c}\text { Aponta a implantação de projetos } \\
\text { que visem ao envolvimento social } \\
\text { de hansenianos como medida de } \\
\text { melhoria da QV. }\end{array}$ \\
\hline Bottene $^{27}$ & $\begin{array}{l}49 \text { pacientes foram avaliados com o } \\
\text { SF-36 e o Dermatology Life Quality } \\
\text { Index. }\end{array}$ & $\begin{array}{l}\text { Não demonstrou déficit na QV em } \\
\text { Paucibacilares. }\end{array}$ & $\begin{array}{l}\text { Indica associação entre QV } \\
\text { e formas de apresentação da } \\
\text { doença. }\end{array}$ \\
\hline Lustosa ${ }^{16}$ & $\begin{array}{l}\text { Estudo observacional com } 107 \\
\text { pacientes } \\
\text { com a aplicação do SF-36 } \\
\text { delineando o perfil sociodemográfico, } \\
\text { clínico e epidemiológico dos } \\
\text { entrevistados. }\end{array}$ & $\begin{array}{c}\text { A correlação entre as variáveis } \\
\text { demonstrou determinantes para } \\
\text { QVRS: } \\
\text { diagnóstico tardio, formas } \\
\text { multibacilares, reações, grau II } \\
\text { de incapacidade no diagnóstico e } \\
\text { preconceito. Acometimento de classes } \\
\text { sociais menos favorecidas em áreas } \\
\text { historicamente endêmicas. }\end{array}$ & $\begin{array}{c}\text { Associação entre o } \\
\text { estabelecimento de formas graves } \\
\text { e incapacitantes de hanseníase } \\
\text { diretamente } \\
\text { relacionadas à baixa QVRS } \\
\text { mesmo no paciente curado. }\end{array}$ \\
\hline
\end{tabular}

* Questionário Abreviado de Qualidade de Vida da OMS

O acompanhamento de pacientes que receberam alta por cura de hanseníase deve ser contínuo, tanto pelas $\mathrm{RH}$ e recidivas, quanto pelos danos psicossociais que os seguem. $\mathrm{O}$ aparecimento destas possui uma relação diretamente proporcional entre reações durante a PQT e as que surgem após o tratamento. ${ }^{1,3,13}$

Os estados reacionais ou reações hansênicas são alterações do sistema imunológico, que se exteriorizam como manifestações inflamatórias agudas e subagudas, que podem ocorrer mais frequentemente nos casos multibacilares. 
Elas podem ocorrer antes, durante ou depois do tratamento com PQT. São classificadas como reação reversa - RR (Tipo 1) e eritema nodoso hansênico - ENH (tipo 2). ${ }^{1,3}$

Os pacientes com reação tipo 1 ou reação reversa, apresentam novas lesões dermatoló $\operatorname{cicas,~infiltrações,~alterações~}$ de cor e edema nas lesões antigas, bem como dor ou espessamento de nervos (neurites). Os pacientes com reação tipo 2, ou eritema nodoso hansênico $(E N H)$ apresentam sintomas gerais como febre, queda do estado geral, astenia, anorexia, artralgias e, muitas vezes, presença de nódulos subcutâneos, vermelhos e dolorosos disseminados. Além disso, pode-se observar neurite isolada, ou seja, neurite sem a presença de lesões de pele provocadas pelas reações. Os portadores de hanseníases podem também apresentar reações mistas, ou seja, reações tipo 1 e 2 ao mesmo tempo e reações tipo eritema polimorfo., ${ }^{1,3}$

Em resultados encontrados por Souza ${ }^{20}$ e Rodrigues ${ }^{21}, 35 \%$ e 23\%, respectivamente, de suas amostras apresentaram $\mathrm{RH}$ em média de um ano após ter recebido a alta. É ressaltada a importância de se conhecer a frequência dos estados reacionais de pacientes que tiveram reação durante a PQT o que permite uma prevenção clínica efetiva, segura e de baixo custo. Por meio da associação do diagnóstico preciso dos tipos de RH e sua evolução é possível definir critérios que decidam ações preventivas clínicas em hanseníase e no seguimento da atenção de saúde com o término da terapia. Pois, existe relação diretamente proporcional entre presença de reações durante e após o tratamento, assim como, as formas clínicas multibacilares apresentam maior frequência de reações durante e após a cura. ${ }^{20,28}$

Martins $^{4}$, ao analisar a percepção de algumas mulheres, ex-portadoras de hanseníase sobre o estigma, preconceito e exclusão que elas sofreram e ainda sofrem, observa os aspectos biopsicossociais da doença, o que destaca a importância de um tratamento não limitado à eliminação do agente etiológico. ${ }^{10,4,29}$ Rodrigues ${ }^{21}$ mostra a relevância de uma ampliação do conceito de cura do paciente de hanseníase no que concerne a cobertura assistencial a esse grupo de pacientes que está fora do registro ativo. A cura, segundo Videres ${ }^{15}$, deve abranger uma reabilitação também social do ex-doente de hanseníase, que vise à supressão de um preconceito injusto e nocivo, enraizado na imagem e história de vida desses indivíduos, com a finalidade de modificar a percepção negativa ou estigmatizante da patologia.

Cercada por associações pejorativas, a hanseníase é carregada de significado cultural, agrega contexto pejorativo, degradante e humilhante aos pacientes em relação as pessoas ignorantes ao processo da doença. ${ }^{30} \mathrm{O}$ portador não possui apenas a doença física, a patologia é percebida também como preconceituosa, uma "metáfora para o mal"; a cura, portanto, deve abranger a eliminação dos preconceitos, a habilitação às pessoas afetadas a defenderem seus direitos, torna-se tão importante como a redução $M$. leprae em sua corrente sanguínea, destaca Nations. ${ }^{31}$

A Hanseníase é caracterizada por perdas acumuladas ao longo do tempo, sejam elas, físicas, sociais ou psicológicas que atingem a qualidade de vida da pessoa enferma. ${ }^{13,22,32,33} \mathrm{O}$ portador torna-se excluído. Problemas como deficiência visual, física de membros inferiores e superiores, alopecia, cicatrizes, lesões hepáticas, renais e outras associadas ao preconceito, são exemplos de deficiências residuais que seguem mesmo após a terapia, o que evidencia a carência de continuidade do tratamento após a eliminação do bacilo. ${ }^{10,15}$

Por suas próprias características, esta doença, exige um contato prolongado com o paciente, tornando o acompanhamento longitudinal, ainda com a alta. A situação do portador inclui dramas de vários tipos relacionados com o tratamento médico, com a situação de pobreza, carência cultural, desagregação familiar, subemprego, além dos problemas psicológicos específicos à doença, relativos à rejeição e à baixa autoestima não se apresentando, apenas, 0 estado da doença como merecedor de atenção, mas todos os outros fatores ligados ao doente e a sua enfermidade. . $3,30,34^{-}$ Quando observados estes aspectos mais os de transmissão e incapacidades a doença se torna complexa, abrangente e o cuidado deve ser, portanto, mais amplo, visto que, a hanseníase causa sofrimento que ultrapassa a dor e o mal-estar estritamente vinculados ao prejuízo físico, com grande impacto social e psicológico. ${ }^{24,35}$

Em estudo feito por Barbosa ${ }^{14}$ foram identificadas lacunas no sistema de saúde de duas cidades cearenses, Sobral e Fortaleza, quando observada à atenção pós-alta, mantendo-se como um desafio para o sistema de saúde, 0 atendimento a estas pessoas. $O$ desenvolvimento de ações multidisciplinares que não apontem apenas para a eliminação, mas também para a prevenção de incapacidades, incentivo à aderência ao tratamento e luta contra o estigma social devem fazer parte do sistema de atenção à saúde destas pessoas a fim de minimizar o impacto da doença na vida destes indivíduos. ${ }^{35}$

Ramos $^{19}$, Souza ${ }^{20}$ e Rodrigues ${ }^{21}$ e Guimarãe ${ }^{36}$, ressaltaram a importância de uma atenção diferenciada a esta população o que permitirá uma prevenção clínica efetiva, segura e de baixo custo na redução de incapacidades provocadas pela doença mesmo depois da alta.

A OMS define qualidade de vida como "A percepção do indivíduo em relação a sua vida no contexto da cultura e do sistema de valores no qual ele vive e em relação a suas metas, expectativas, modelos e preocupações". ${ }^{37}$ Halioua ${ }^{38}$ 
aponta-a como um conceito amplo que abrange saúde física, estado psicológico, nível de independência, relações sociais, opiniões, integração com o meio ambiente dentre outros fatores.

A partir destes conceitos, Savassi ${ }^{22}$ analisando QV em ex-portadores de hanseníase, observou que o paciente 0 qual possui expostas suas sequelas tem pior qualidade de vida. Outra correlação observada, como consequência do comprometimento provocado pela doença, foi de que a maioria não trabalha; o que vem a caracterizar uma má reinserção social dos mesmos. As atividades físicas foram apontadas como um meio de ajudá-los nesta reinserção, pois, colaborou para que estes possam lidar melhor com os aspectos sociais e pessoais, fazendo-os sentir novamente incluídos na sociedade, agindo positivamente na QV. ${ }^{22,23}$

Em pesquisas feitas por Savassi ${ }^{22}$ e Videres ${ }^{15}$, relatos de portadores de hanseníase evidenciaram a discriminação e a rejeição que sofreram por parte de pessoas próximas, como vizinhos, conhecidos e até de familiares. 0 preconceito foi relacionado ao conhecimento do diagnóstico por parte dos contatos destes portadores. Os portadores da doença mantêm um comportamento de fuga dos contatos sociais e apresentam seus próprios preconceitos em relação à moléstia, rejeitam a si mesmos, não se aceitam como portadores da doença e isolam-se de seu grupo social. ${ }^{2}$

Quanto à atitude do paciente em contar sobre sua doença aos familiares e amigos há um elevado grau de estigma pessoal e social entre os pacientes, diante da enfermidade, da família e amigos para com os pacientes, reafirmando uma postura de autoflagelo descrita por Simões ${ }^{39}$ e Martins. $^{23}$

Dentre os principais determinantes para QV relacionada à hanseníase são apontados: diagnóstico tardio, formas multibacilares, reações hansênicas, grau II de incapacidade no diagnóstico, preconceito, a capacidade de trabalho após o tratamento, as limitações de atividades, a baixa renda e à escolaridade. ${ }^{16,17,32,34,40,41}$

A educação influencia intensamente na cadeia de transmissão da doença e estigma gerado pela falta de compreensão da população. ${ }^{13}$ Além disso, o difícil acesso a informações e serviços de saúde podem propiciar o surgimento de casos mais graves em função do diagnóstico tardio. ${ }^{42}$ Martins ${ }^{23}$ observou que quanto menor a escolaridade e a renda familiar, maior é o comprometimento da QV do paciente com hanseníase, sobretudo, no que diz respeito às relações sociais e a aceitação da doença pelos outros e pelo próprio portador. A relação entre QV em hansenianos e escolaridade foi também analisada por Joseph ${ }^{25}$ destacando-se a proporcionalidade entre as variáveis.

Rafael ${ }^{13}$, ao correlacionar graus de incapacidade com escores de Escalas de Participação (EP) e SALSA (Screening of Activity Limitation and Safety Awareness) em portadores e ex-portadores de hanseníase mostra que tal patologia atinge uma população economicamente ativa, com consequente prejuízo a força de trabalho provocando uma dificuldade de provir seu sustento e o de sua família produzindo forte impacto socioeconômico.

O uso de ferramentas para a atenção integral aos portadores, inclusive após a alta de hanseníase como a escala SALSA, para a limitação da atividade funcional/consciência de risco e a Escala de Participação são destacadas na literatura como eficientes para uma maior compreensão e atuação nesta população. . $7,43,13^{-13}$

Brouwers ${ }^{26}$ defende o desenvolvimento de estratégias que visem ao diagnóstico precoce, prevenção de incapacidades, e reabilitação social para melhorar a qualidade de vida das pessoas acometidas, além de um direcionamento para reinserção na comunidade e no mercado de trabalho por meio de programas geradores de renda.

A hanseníase causa sofrimento e impactos sobre a $Q V$ de seus afetados que ultrapassam a dor e o mal-estar estritamente vinculados ao prejuízo físico, com grande impacto social e psicológico, justificando tanto avanços para abordagem multidisciplinar ao paciente quanto à necessidade de ações de saúde que visem ao controle da doença ${ }^{32,27}$ Avaliar a QV dos portadores e ex-portadores de hanseníase mostra-se de fundamental importância para o desenvolvimento de práticas assistenciais e políticas publicas que tenham como objetivos a promoção de saúde integral e a prevenção da doença e que repercutam positivamente na esfera psico-sócio-cultural da enfermidade. ${ }^{23}$

\section{Considerações Finais}

De acordo com a definição da OMS ${ }^{44}$, a saúde é compreendida como completo bem-estar biopsicossocial. Portanto, a atenção à saúde destas pessoas não se esgota com o término do esquema de PQT, danos permanentes as acompanham comprometendo sua qualidade de vida.

A ampliação do conceito de cura para a referida patologia será alcançada com o estabelecimento de estratégias de reinserção destas pessoas à comunidade visando à educação popular para eliminação do estigma que cerca 
a doença e provoca danos severos aos seus portadores a fim de restaurar sua saúde por completo. A inclusão de medidas assistenciais à população em pós-alta de hanseníase é indispensável para o cumprimento de um dos princípios norteadores do Sistema Único de Saúde (SUS), o de integralidade.

\section{Referências Bibliográficas}

1. Brasil, Ministério da Saúde. Secretaria de Políticas de Saúde. Departamento de Atenção Básica. Guia para o Controle da hanseníase. Brasília, 2002.

2. Eidt LM. Ser hanseniano: sentimentos e vivências. Hansen. Int. 2004;29(1):21-7.

3. Organização Mundial de Saúde (OMS). Estratégia global aprimorada para redução adicional da carga da hanseníase: 2011-2015: diretrizes operacionais (atualizadas). /Organização Pan-Americana da Saúde. Brasília, 2010.

4. Martins PV, Caponi S. Hanseníase, exclusão e preconceito: histórias de vida de mulheres em Santa Catarina. Ciência \& Saúde Coletiva 2010;15(Supl.1):1047-54.

5. World Health Organization (WHO). The Final Push Toward Elimination of Leprosy: Strategic Plan 2000-2005. Geneva: WHO/CDC/CPE/CEE/, 2000

6. Brasil, Ministério da Saúde. Secretaria de Vigilância em Saúde. Departamento de Vigilância em Doenças Transmissíveis. Plano integrado de ações estratégicas de eliminação da hanseníase, filariose, esquistossomose e oncocercose como problema de saúde pública, tracoma como causa de cegueira e controle das geohelmintíases: plano de ação 2011-2015 / Ministério da Saúde, Secretaria de Vigilância em Saúde, Departamento de Vigilância em Doenças Transmissíveis. Brasília, 2012.

7. Martelli CMT, Stefani MMA, Penna GO, Andrade ALSS. Endemias e epidemias brasileiras, desafios e perspectivas de investigação científica: Hanseníase. Rev. Bras. Epidemiol. 2002;5(3):273-285

8. World Health Organization (WHO). Weekly Epidemiological Record. September, 86th year;(36):389-400, 2011.

9. Coelho AR, O Sujeito diante da Hanseníase. Pesq e Prát Psicossoc Fev 2008;2(2):364-372.

10. Lira CV, Catrib AMF, Nations MK, Lira RCM. A hanseníase como etno-enfermidade: em busca de um novo paradigma de cuidado. Hansen int 2005;30(2):185-194.

11. Balaiarde KS, O Estigma da Hanseníase: Relato de uma experiência em grupo com pessoas portadoras. Hansen Int 2007;32(1):27-36.

12. Bittencuort LP, Carmo AC, Leão AMM, Clos AC. Estigma: Percepções Sociais Reveladas por Pessoas Acometidas por Hanseníase. Rev enferm UERJ abr/jun 2010;18(2):185-90.

13. Rafael AC, Pacientes em tratamento pós-alta em hanseníase: estudo comparativo entre os graus de incapacidades preconizados pelo Ministério da Saúde correlacionando-os com as escalas SALSA e Participação Social. Brasília: Universidade de Brasília, Departamento de Ciências Médicas, 2009.

14. Barbosa JC , Pós-alta em hanseníase no Ceará: olhares sobre políticas rede de atenção à saúde, limitação funcional, de atividades e participação social das pessoas atingidas. São Paulo: Universidade de São Paulo, Faculdade de Saúde Pública, 2009. 15. Videres ARN. Trajetória de vida de ex-portadores de hanseníase com histórico asilar. Universidade Federal do Rio Grande do Norte, Programa de Pós-Graduação em Enfermagem, 2010.

16. Lustosa AA, Nogueira LT, Pedrosa JIS, Teles JBM, Viriato C. The impact of leprosy on health-related quality of life. Rev Soc Bras Med Trop set-out 2011;44(5):621-626.

17. Ikehara E, Nardi SMT, Ferrigno ISV, Pedro HSP, Paschoal VD. Escala Salsa e grau de Incapacidades da Organização Mundial de Saúde: avaliação da limitação de atividades e deficiência na hanseníase. Acta Fisiatr 2010;17(4):169 - 174. 18. Aquino DMC, Santos JS, Costa JML. Avaliação do programa de controle da hanseníase em um município hiperendêmico do Estado do Maranhão, Brasil, 1991-1995. Cad. Saúde Pública 2003 jan-fev; 19(1): 119-125.

19. Ramos JMH. Souto FJD. Incapacidade pós-tratamento em pacientes hansenianos em Várzea Grande, Estado de Mato Grosso. Rev Soc Bras Med Trop mai-jun 2010;43(3):293-297.

20. Souza LWF. Reações hansênicas em pacientes em alta por cura pela poliquimioterapia. Rev Soc Bras Med Trop novdez 2010;43(6):737-739.

21. Rodrigues ALP, Almeida AP, Rodrigues BF, Pinheiro CA, Borges DS, Mendonça MLH, et al. Ocorrência de reações em pacientes pós-alta por cura de hanseníase: subsídios para implementação de um programa de atenção específica. Hansen Int 2000;25111: 7-16. 
22. Savassi LCM. Hanseníase: políticas públicas e qualidade de vida de pacientes e seus cuidadores. Fundação Oswaldo Cruz, Programa de Pós-graduação em Ciências da Saúde, 2010.

23. Martins MA, Qualidade de Vida em Portadores de Hanseníase 2009. Campo Grande/MS: Universidade Católica Dom Bosco, Mestrado em Psicologia, 2009.

24. Paschoal VD, Soler ZASG. Um sistema de cores na caracterização biopsicossocial do portador de hanseníase reacional. Hansen Int 1999;24(1):21-31.

25. Joseph GA, Rao PSSS. Impact of leprosy on the quality of life. Bulletin of the World Health Organization, 1999;77(6). 26. Brouwers C, Brakel WV, Cornielje H. Quality of life, perceived stigma, activity and participation of people with leprosyrelated disabilities in south-east Nepal. Disability, CBR \& Inclusive Development 2011;22(1).

27. Bottene IMC. Qualidade de vida em pacientes com hanseníase paucibacilar. Universidade de São Paulo, Faculdade de Medicina, 2011.

28. Brito MFM, Ximenes RAA, Gallo MEN, Bührer-Sékula S. Association between leprosy reactions after treatment and bacterial load evaluated using anti PGL-I serology and bacilloscopy. Rev Soc Bras de Med Trop 2008;41(Supl II):67-72.

29. Rafferty J, Curing the stigma of leprosy. Lepr Rev 2005;76:119-126.

30. Lenita B, Claro L, editores. Hanseníase: Representações sobre a Doença. Rio de Janeiro: Editora Fiocruz, 1995, 195p. 31. Nations MK, Lira GV, Catrib AMF. Stigma, deforming metaphors and patients'moral experience of multibacillary leprosy in Sobral, Ceará State, Brazil. Cad Saúde Pública jun 2009; 25(6):1215-1224.

32. Martins BDL, Torres FN, Oliveira MLW. Impacto na qualidade de vida em pacientes com hanseníase: correlação do Dermatology Life Quality Indexcom diversas variáveis relacionadas à doença. An Bras Dermatol 2008;83(1):39-43.

33. Quaggio CMP. Hanseníase: Qualidade de vida dos moradores da Área Social do Instituto Lauro de Souza Lima. Programa de Pós-Graduação em Infecções e Saúde Pública da Coordenação de Controle das Doenças da Secretaria do Estado da Saúde do Estado de São Paulo, 2005.

34. Nunes JM, Oliveira EM, Vieira NFC, Hanseníase: conhecimentos e mudanças na vida das pessoas acometidas. Ciência \& Saúde Coletiva 2011;16(Supl.1):1311-1318.

35. Costa MD, Costa RD, Costa AMDD, Terra FS, Lyon S, Antunes CMF. Assessment of quality of life of patients with leprosy reactional states treated in a dermatology reference center. An Bras Dermatol. 2012;87(1):26-35.

36. Guimarães LS, Bandeira SS, Leão MRN, Barreto JG, Denis VGF, Rosa PS, Frade MAC, Salgado CG. Incapacidade pós-alta em pessoas atingidas pela hanseníase no estado do Pará. $12^{\circ}$ Congresso Brasileiro de Hansenologia / Congresso Regional da ILA - Américas; Maceió - Alagoas - Brazil; Hansen Int 2011;36(1):236.

37. The WHOQOL Group. The World Health Organization Quality of Life Assessment (WOQOL): Position Paper From the World Health Organization, Soc Sci Med 1995; 41:1403-10.

38. Halioua B, Beumont MG, Lunel F. Quality of Life in Dermatology. Int J Dermatol 2000;39:801-6.

39. Simões MJS, Dellelo D. Estudo do Comportamento Social dos Pacientes de Hanseníase do Município de São Carlos - SP. Rev Esp para a Saúde dez 2005;7(1):10-15

40. Duarte MTC, Ayres JA, Simonetti JP. Perfil socioeconômico e demográfico de portadores de hanseníase atendidos em consulta de enfermagem. Rev Latino-Am Enfermagem 2007 setembro-outubro; 15 (esp).

41. Paschoal VDA, Nardi SMT, Cury MRCO, Lombardi C, Virmond MCL, Silva RMDN, et al. Criação de banco de dados para sustentação da pós-eliminação em hanseníase. Ciênc. \& Saúde Col. 2011;16(Supl.1):1201-1210.

42. Pinto RA, Maia HF, Silva MAF, Marback M. Perfil Clínico e Epidemiológico dos Pacientes Notificados com Hanseníase em um Hospital Especializado em Salvador, Bahia. Rev. B. S. Publica Miolo. out/dez 2010;34(4):906-918.

43. Barbosa JC, Jr ANR, Alencar MJF, Castro CGJ. Pós-alta em Hanseníase no Ceará: limitação da atividade funcional, consciência de risco e participação social. Rev Bras Enferm 2008;61(esp):727-33.

44. OMS (Organização Mundial de Saúde) 1946. Constituição. Disponível em <http://www.onuportugal.pt/"oms.doc>. Acesso em 10 de fevereiro de 2003. 


\section{Mara Dayanne Alves Ribeiro}

Endereço para correspondência - Avenida São Sebastião, n 2819. Bairro Reis Velloso, CEP: 64240-000, Parnaiba, PI, Brasil

E-mail: mara_dayanne2@hotmail.com

Lattes: http://lattes.cnpq.br/8526791971124084

Sabrynna Brito Oliveira - sabrynnabrito@gmail.com

Marcelo de Carvalho Filgueiras - professormarcelo@ufpi.edu.br

Enviado em 17 de abril de 2013.

Aceito em 07 de julho de 2014. 